\title{
Approche Ethno-Vétérinaire Des Plantes Médicinales Utilisées Dans La Région De Sidi Bel Abbes- Algérie
}

\author{
Merazi Yahya \\ Hammadi Kheira \\ Fedoul Firdaous Faiza
}

Laboratoire de pharmacognosie Api phytothérapie ;

Département de Biologie ; Faculté SNV ;Université de Mostaganem-Algérie

doi: 10.19044/esj.2016.v12n18p218 URL:http://dx.doi.org/10.19044/esj.2016.v12n18p218

\begin{abstract}
The control of the health risk affecting human health and animal remains a major problem in Algeria and in the world. The high use of antibiotics led to the antibiotic resistance, which is observed in farmed animals since these last decades. In this work is an approach to the traditional remedies in poultry farming in veterinary medicine. A survey was conducted with 60 farmers in the region of Sidi Bel Abbes of the West- Algeria.

This study was designed to understand the way to treat diseases and the traditional knowledge of breeders on the plants, which are derived from the tradition of treating farm animals in Algeria. No work has been done before at this region for this reason, we have therefore preferred a survey for this study. A questionnaire has been developed specifically to this effect focused on: Profile of the respondents, characteristics of the medicinal plants, traditional treatments. Our results have revealed that $85 \%$ of women have a know quite important treating plants, $75 \%$ respondents aged over 60 years and $55 \%$ of illiterate practice use the medicinal plants to treat their animals.

The most used plants are Thymus capitatus 20\% followed by thymus vulgaris $15 \%$, their use is dominated by decoction $31.67 \%$; the sheets are more used part of the plants $26.67 \%$ the forest $30 \%$ because of the forest source at this region study, $91.67 \%$ of spontaneous plants are harvested, the mode of Oral admission uses are respectively $70 \%, 53.33 \%$. $25 \%$ in a sheeps and $20 \%$ in chickens where they undergo a basic treatment of medicinal plants.
\end{abstract}

Keywords: Survey, medicinal plants, ethno-veterinarian

\section{Résumé}

La maîtrise du risque sanitaire touchant la santé humaine et animal reste un problème majeur en Algérie et dans le monde entier.la forte 
utilisation des antibiotiques conduits à l'antibiorésistance, qui s'observe chez les animaux d'élevage depuis ces dernières décennies. Dans ce cadre, une recherche d'approcher les remèdes traditionnelles en aviculture en médecine vétérinaire. Une enquête a été réalisée avec 60 éleveurs dans la région de Sidi Bel Abbes de l'Ouest- Algérie. Cette étude visait à mieux comprendre la manière dont se traité les maladies et les savoirs traditionnels des éleveurs sur les plantes, qui sont issus de la tradition orale. Pour cette raison, nous avons donc privilégié l'enquête orale. Un questionnaire a été développé spécifiquement à cet effet portaient sur: profile des enquêtés, caractéristiques des plantes médicinales, traitements traditionnelles.nos résultats ont révélés que $85 \%$ des femmes présentent un savoir assez important, 75\% enquêtés âgés plus de 60 ans sont des connaisseurs de la matière de plante médicinale, alors $55 \%$ des analphabète pratique la médecine douce. Les plantes les plus utilisées sont Thymus capitatus 20\% suivie de Thymus vulgaris 15\%, leurs utilisation est dominaient par décoction $31.67 \%$, leurs partie utilisée est les feuilles $26.67 \%$, leurs source est la forêt 30\%, 91.67\% des plantes spontanées sont récoltées, le mode d'administration orale et L'efficacité est respectivement 70\%, 53.33\%. 25\% des moutons et $20 \%$ des poulets subit un traitement à base des plantes médicinales.Les éleveurs sont loin d'être équipé par un matériel de diagnostiquer leurs élevages en cas de pathologie.

Mots clés : Enquête ; plantes médicinales ; ethno-vétérinaire

\section{Introduction}

La médecine ethno-vétérinaire (MEV) est un terme scientifique pour les soins de santé animale traditionnelle qui englobe les connaissances, les compétences, les méthodes, les pratiques et les croyances au sujet des soins de santé des animaux trouvés parmi les membres de la communauté(McCorkle, 1986). Elle s’occupe de la prévention des maladies, la préparation d'une vaste pharmacopée et la lutte contre les pathologies (virales, bactériennes, parasitaires ...)

Au lendemain de l'indépendance, la production avicole dans sa quasitotalité se reposait essentiellement sur l'élevage familial et quelques exploitations et unités de petite envergure (Lyes \& Kirouani, 2015).

L'intérêt pour les plantes médicinales à usage vétérinaire a récemment augmenté, du fait ne cause pas en premier degré des problèmes de résistance et d'effets secondaires néfaste à l'environnement que celle apporté par les médicaments non naturelle.

Vue la facilité à préparer et à administrer les médicaments à base de plantes et sa gratuité, la médecine ethno-vétérinaire occupe une place non négligeable dans les pratiques des éleveurs Algériens. 
Certaines plantes sont en train de disparaître à la suite de l'accroissement de la population, des méthodes de récolte non durables et d'autres problèmes environnementaux. C'est pourquoi il est important de collecter les remèdes ethno-vétérinaires. Ces derniers sont transmis oralement de génération en génération.

Afin d'empêcher nos remèdes de disparaitre avec leur plantes et avec les personnes âgés, et dans le but d'améliorer les informations sur les applications thérapeutiques et traditionnelles en médecine vétérinaire en aviculture, et assuré un lien entre les pratiques ancestrales et la médecine conventionnel, une enquête sur terrain et auprès des éleveurs traditionnels de poules a été réalisée.

\section{Matériel et Methodes \\ Enquête \\ Questionnaire}

Notre étude visait à mieux comprendre la manière dont se traité les maladies et les savoirs traditionnels des agriculteurs et les éleveurs de notre région d'étude (ouest d'algerie) par les plantes, qui sont issus de la tradition orale. Pour cette raison, nous avons donc privilégié l'enquête orale.

Les informations ont été obtenues à travers des entrevues ethnobotaniques avec des personnes nées ou ayant vécu longtemps dans la commune (Mehdioui \& Kahouadji, 2007) de Tilmouni, HassiDahou, Belarbi, Oued Sefioune, Tenira et Amarna auprès des trois forêts Moksi, Bouhriz et Tenira (wilaya de sidi bel abbés région de l'ouest d'Algérie)

Douze fermes dans la région d'étude ont été visitée afin d'inspirer les paysans de participer a notre enquête. Ils se sont imprégnés des réalités de l'activité avicole .On a ciblé un échantillon d'hommes et femmes.

La localisation des différents milieux d'enquêtes ethnobotaniques et de relevés floristiques, dans la zone étudiée a été repérée par les techniques d'échantillonnage «stratifié probabiliste»(Kahouadji, 1986).

Dans ce travail, l'échantillon est divisé en 6 strates (Tableau 1). En procédant par un échantillonnage aléatoire simple, des échantillons de nombres restreints (10 personnes) sont formés pour chacune des 6 strates qui sont mis ensemble pour constituer l'échantillon global (60 personnes).

Tableau 1.Repartirion des enquêtes en fonction des strates.

\begin{tabular}{ccc}
\hline Strates & Nom des strates & Nombre d'enquêtes \\
\hline Strate1 & Tilmouni & 10 \\
Strate2 & Hassi Dahou & 10 \\
Strate3 & Belarbi & 10 \\
Strate4 & Oued Sefioune & 10 \\
Strate5 & Tenira & 10 \\
Strate6 & Amarna & 10 \\
& Echantillon & 60 \\
\hline
\end{tabular}


La localisation de ces six communes peut être lue sur la carte réalisée par un capteur d'écran (Figure 1). Ces localités ont été choisies pour leur facilité d'accès et du fait que un grand nombre habitas possèdent un effectif en cheptel animal important.

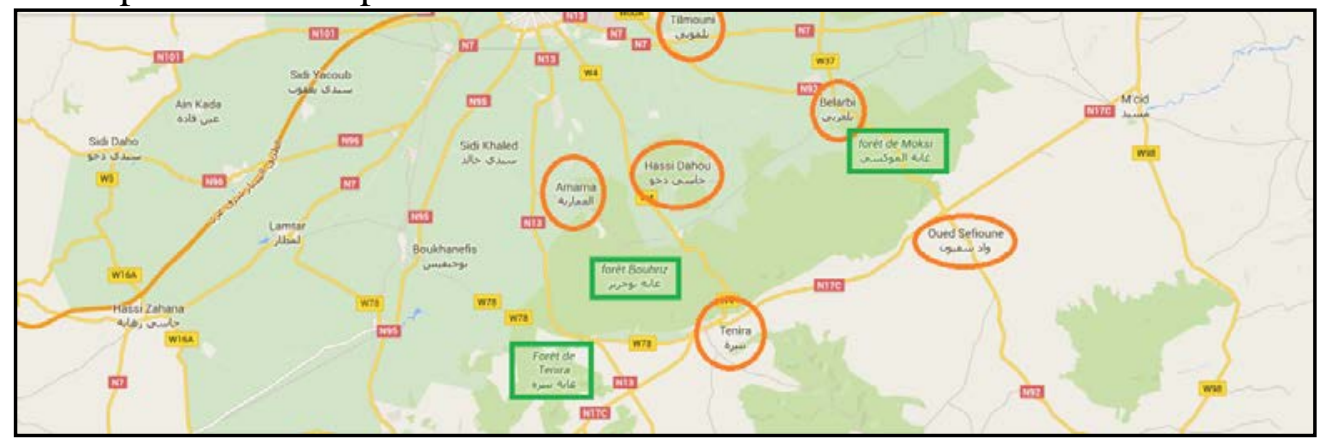

Figure 1: Répartition des zones d'enquêtes en fonction des strates.(Google earth)

Un questionnaire a été développé spécifiquement portaient sur trois axes:

AXE1 pour chaque enquêté : sexe, âge, niveau scolaire ... ;

AXE2 pour chaque plante : la Parties utilisées, la forme d'utilisation, les plantes utilisées (nom local), source de provenance ... etc ;

AXE3 concernent le taux et les symptômes qui touchent les animaux d'élevages.

À l'aide d'une fiche remplie par interrogation orale, les informations sont enregistrées immédiatement afin d'être traitées ultérieurement.

\section{Choix et description de la zone d'étude}

Le choix de la zone de végétation repose sur une homogénéité du couvert végétal, outre à La région d'étude qui est devenue un pôle de développement en élevage avicole familial (Figure 2).

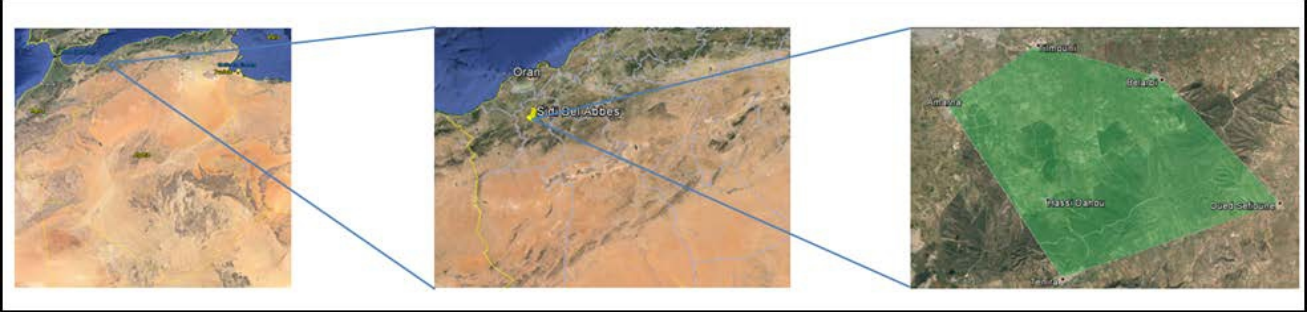

Figure 2: La région d'étude sidi bel abbés ouest d’Algérie

Des relevés floristiques ont été réalisés pendant la période de floraison de la végétation (Mars-avril 2014), Tenant compte les conseils d'éleveurs sur la source de plantes médicinales (Tableau 2). 
Tableau 2 : Données géographiques de la zone d'étude

\begin{tabular}{|c|c|c|}
\hline Cites d'études & Latitude & Longitude \\
\hline Tilmouni & 35.1749675 & -0.54843195 \\
\hline Amarna & 35.1366774 & -0.62325599 \\
\hline Tenira & 35.0204525 & -0.53229705 \\
\hline Oued Sefioune & 35.0672641 & -0.35718198 \\
\hline Belarbi & 35.1511727 & -0.45021029 \\
\hline
\end{tabular}

\section{Échantillonnage}

Le matériel végétal est constitué des parties aériennes des plantes, qui sont acheminée au laboratoire à l'état frais et à l'abri de la lumière et l'humidité. En respectant les conditions et période d'échantillonnage. Elle tient compte de la vigueur et de l'état sanitaire des plantes mais aussi des conditions du milieu. La cueillette doit se faire par beau temps, sans vent et sans pluie (Jean \& Jiri, 1983) .

Les plantes médicinales sont manipulées avec beaucoup de précaution après la récolte, pour éviter que ses substances chimiques actives qui exercent une action thérapeutique ne disparaissent. L’identification taxonomique des espèces a été réalisée ultérieurement à l'aide de la littérature

\section{Résultats et Discussion}

\section{AXE 1 : Profil des enquêtes}

\section{Utilisation des plantes selon le sexe, l'âge et le niveau scolaire}

Sexe : Dans la région d'enquête les femmes présentent un savoir assez important par rapport aux hommes sur l'utilisation des plantes médicinales $85 \%$ pour $15 \%$ d’homme (Tableau 2). Ces résultats confirment d'autres travaux ethnobotaniques réalisé Mehdioui et Kahouadji (2007) dans la forêt d'Amsittène (Province d'Essaouira au Maroc) et Benkhnigue et al (2010) dans la région de Mechraâ Bel Ksiri (Région du Gharb au Maroc) et El Hafian et al (2014) d'Agadir-Ida-Outanane Maroc et Bouallala et al (2014) dans la région du Souf (Algérie) ou les femmes sont plus détentrices du savoir phytothérapique traditionnel.

Age : Les âgés plus de 60 ans ont montrés un plus grand intérêt dans leur connaissance des usages et des propriétés des plantes médicinales75\% par rapport aux générations mois de 60 ans avec un pourcentage 25\% (Tableau 3). Ceci a été confirmé par une étude Daoudi Amine et al (2015).

La transmission de cette connaissance est en danger actuellement parce qu'elle n'est pas toujours assurée (Anyinam, 1995; Benkhnigue et al., 2010; El Hafian, Benlandini, Elyacoubi, Zidane, \& Rochdi, 2014; Mehdioui \& Kahouadji, 2007).

Ces résultats confirment effectivement que les pratiques de guérison traditionnelles est en disparition 
Niveau scolaire : Nos résultats confirment que la pratique traditionnelle est inversement proportionnelle au niveau d'instruction avancée $55 \%$ sont analphabète ,26.66\% ont un niveau primaire, $15 \%$ ont un niveau moyen, 3.33\% ont arrivés au lycée (Tableau 2), en effet l'apparition des pratiques modernes empêcha les jeunes générations à utiliser les connaissances et pratiques de leurs ancêtres.

Ces résultats confirment d'autres travaux Mehdioui \& Kahouadji (2007) et El Hafian et al (2014). Ce qui est contrairement obtenu par Bouallala et al (2014) qui montre que les personnes ayant le niveau secondaire utilisent beaucoup les plantes médicinales par rapport aux personnes analphabètes.

Tableau 3 : Profil général des enquêtés

\begin{tabular}{|c|c|c|}
\hline \multicolumn{2}{|c|}{ Caractéristiques } & Pourcentage \\
\hline \multirow{2}{*}{ Age : } & 0 à 19 & $0 \%$ \\
\cline { 2 - 3 } & 20 à 39 & $5 \%$ \\
\cline { 2 - 3 } & 40 à 59 & $20 \%$ \\
\cline { 2 - 3 } & plus de 60 & $75 \%$ \\
\hline \multirow{2}{*}{ Sexe : } & Femme & $85 \%$ \\
\hline \multirow{2}{*}{ Niveau scolaire : } & Homme & $15 \%$ \\
\hline & Analphabète & $55 \%$ \\
\cline { 2 - 3 } & Primaire & $26.67 \%$ \\
\cline { 2 - 3 } & moyen & $15 \%$ \\
\cline { 2 - 3 } & lycée & $3.33 \%$ \\
\hline
\end{tabular}

\section{AXE 2 : Information sur les plantes}

\section{Les parties utilisées}

Les feuilles sont les parties les plus utilisées avec un taux de (26.67\%); suivies par les tiges feuillée (21.67 \%), la partie aérienne et plante entière partage (15\%), les fruits (13.33\%), et la partie souterraine (8.33\%) (Figure 3).

Nos résultats confirment que les feuilles possèdent un intérêt important en médecine traditionnelle, cela n'est pas le cas de (Lulekal, Asfaw, Kelbessa, \& Van Damme, 2014) dans l'étude plantes ethnovétérinaires de Ankober District, Shewa Nord, région d'Amhara, en Ethiopie, confirment que les racines sont les plus utilisées puis des mélanges de feuilles et de racines , et (Kpodékon et al., 2015) dans l'étude pathologies virales dominantes chez l'animal extensive et semi-intensive, confirment les écorces sont les plus utilisées suivie par les feuilles

Selon (Ogni et al., 2014; SALHI, FADLI, ZIDANE, \& DOUIRA, 2010), les organes les plus utilisés sont celle les plus facile à récoltés. Confirment aussi le travail de (Chehma \& Djebar, 2008; Hammadi, Ahmed, 
Boudjethia, Boukhalfa, \& Djebli, 2015). Concernant la partie aérienne est très utilisée.

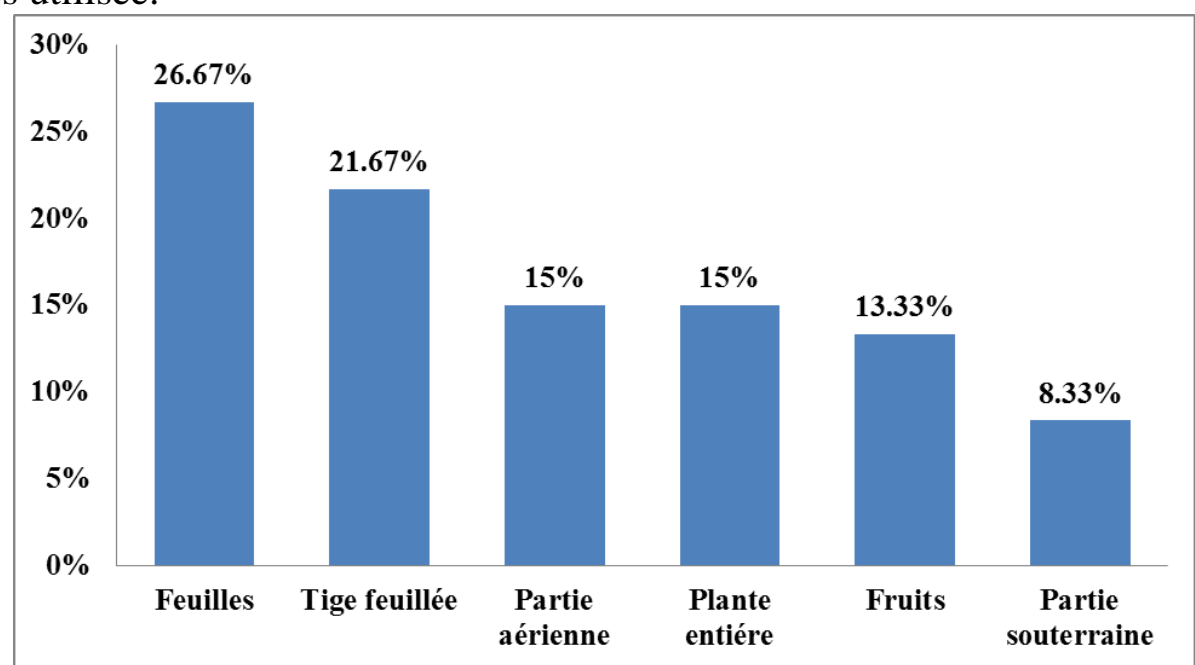

Figure 3 : Répartition des différentes parties utilisées des plantes médicinales

\section{La forme d'utilisation}

L'analyse de l'information collectée montre que la préparation des remèdes traditionnelle à base de plante médicinale peut être par décoction $31.67 \%$, poudre $18.33 \%$, cataplasme $16.67 \%$, infusion $15 \%$, fumigation $13.33 \%$, et autre préparation qui ne dépasse pas les 5\% (Figure 4).

Nos résultats sont autour de celle obtenus par divers auteurs (Benkhnigue et al., 2010; Bouallala, Bradai, \& Abid, 2014; Daoudi , Bachiri , Bammou , Ibijbijen , \& Nassiri 2015; El Hafian et al., 2014).

Malgré que (Benkhnigue et al., 2010; Tahri, El Basti, Zidane, Rochdi, \& Douira, 2012) confirment que infusion, cataplasme, décoction occupent une place importante dans les remèdes de nos ancêtre. Cela repose sur la seule façon, facile, rapide et efficace de tirer le secret des plantes.

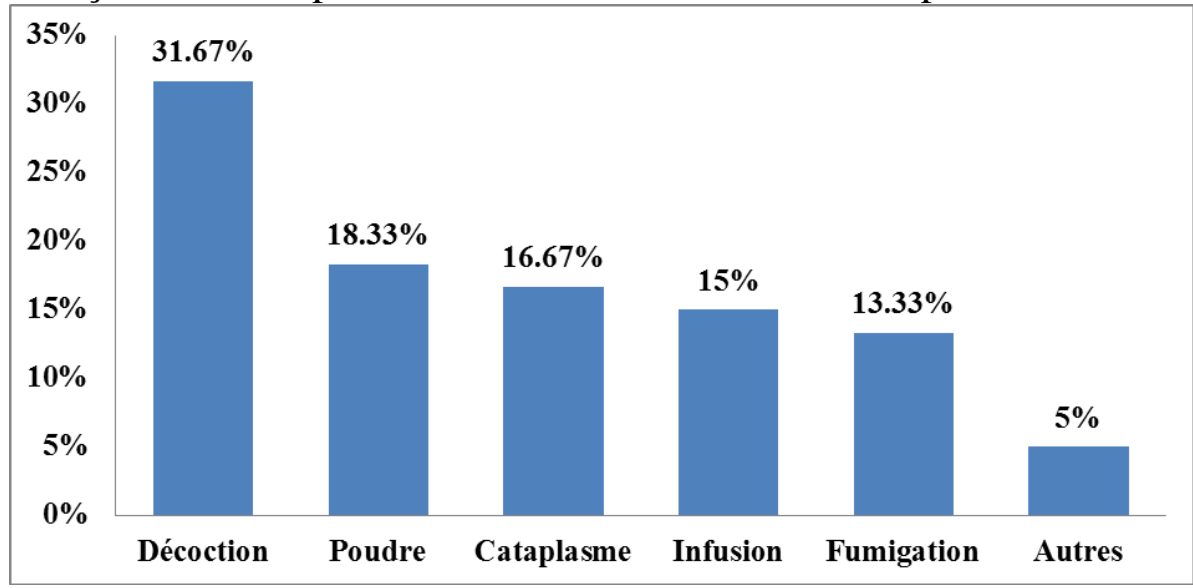

Figure 4 : Répartition des différents modes de préparation des plantes médicinales 


\section{Les plantes utilisées}

Au cours de cette étude, sept (7) plantes étaient ciblée pour des analyses ultérieures.

Thym capitatus 20\%, Thymus vulgaris $15 \%$,la verveine $13.33 \%$, le romarin $11.67 \%$, l'armoise blanche $10 \%$, la sauge $8.33 \%$,la rue $5 \%$,les clous de girofle3.33\% ,autres13.33\% (Figure 5).

Les mêmes résultats ont été confirmés par Hammadi et al (2015)

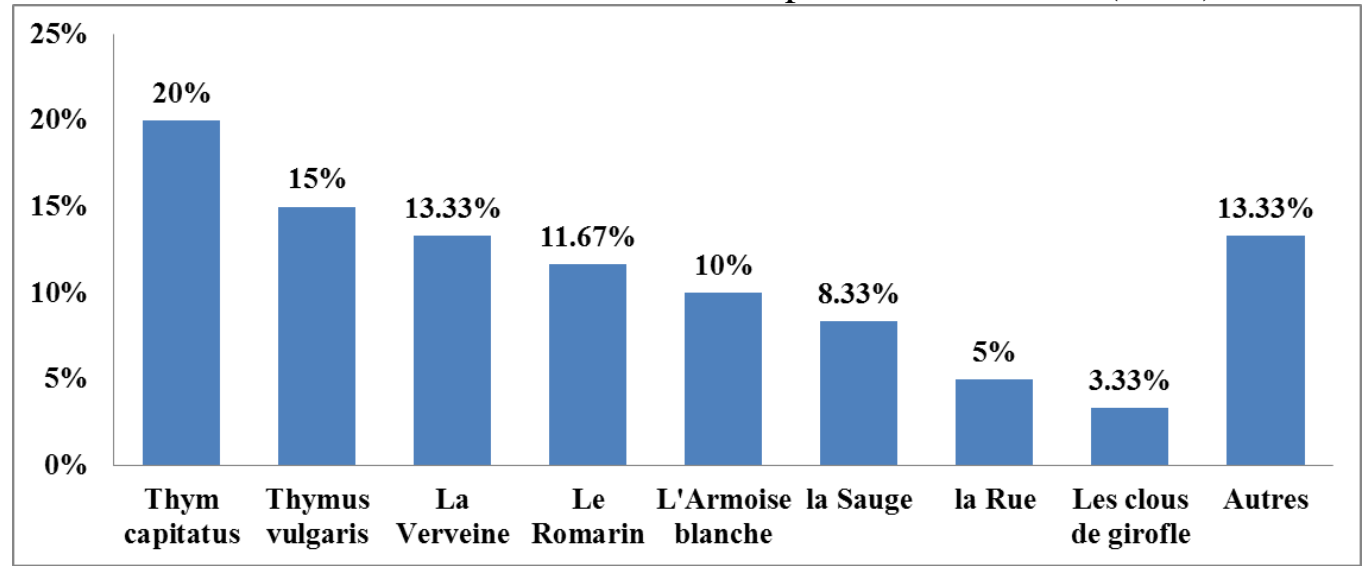

Figure 5 : Répartition de la fréquence d'utilisation des espèces des plantes médicinales fréquemment utilisées.

\section{La source de provenance des plantes}

36.66\% des plantes proviennent de marché publique, 30\% de foret, $6.66 \%$ sont cultivé et $26.66 \%$ reste de source mal connu (Figure 6).

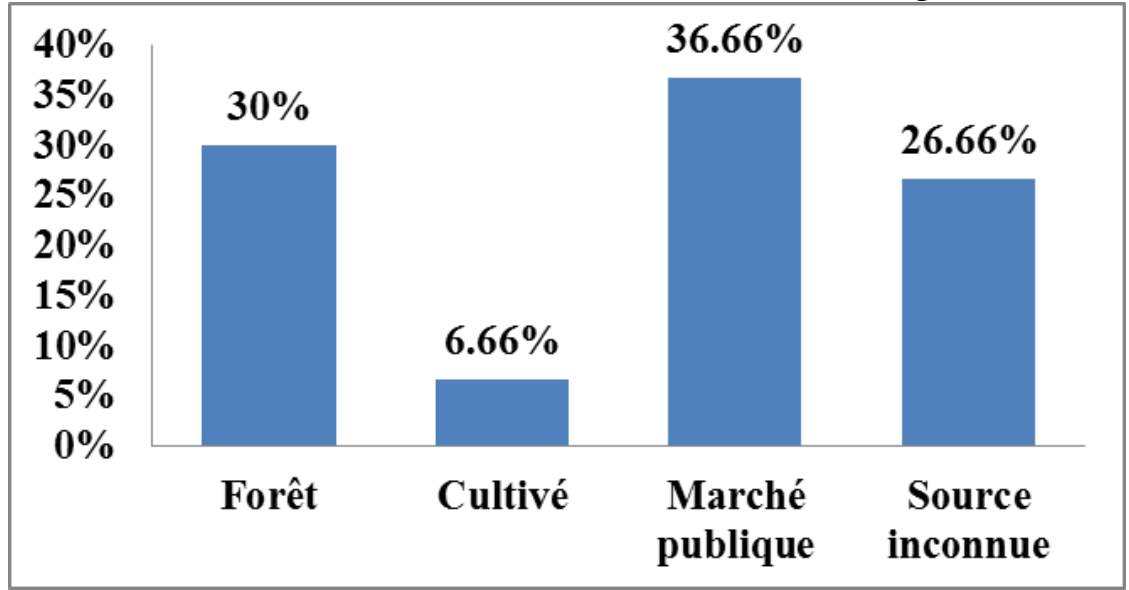

Figure 6 : Répartition de la fréquence des espèces médicinales selon leurs sources de provenance.

\section{Le type de plante}

91.67\% des plantes spontanées sont récoltées dans la région d’étude et d'autres régions comme sud de sidi bel abbés, alors que $8.33 \%$ des espèces 
sont cultivées (Figure 7). Parmi ces dernières, on a les clous de girofle, sont cultivées autour de quelques habitations. Ces résultats sont identiques à celle obtenu par Mehdioui et Kahouadji, (2007)

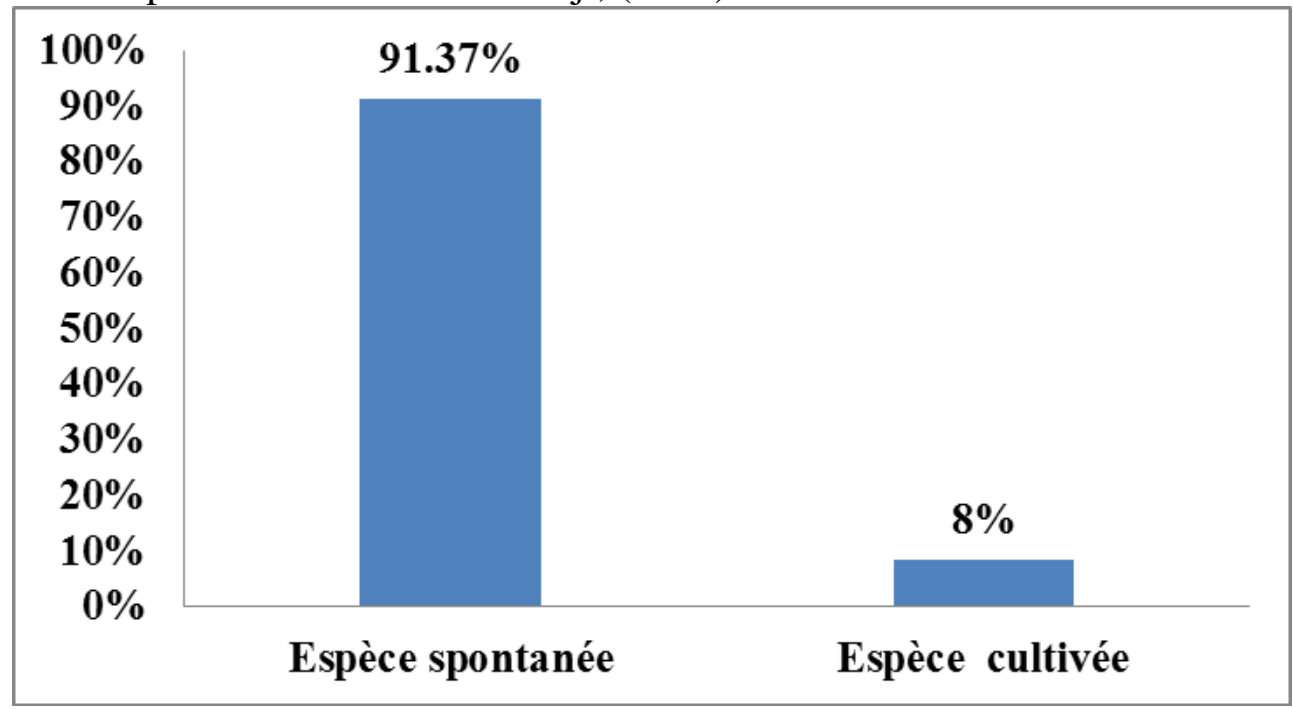

Figure 7 : Répartition de la fréquence des espèces médicinales selon le mode de culture

\section{Mode d'administration}

La voie orale occupe un taux de cumule $70 \%$, fumigation, $15 \%$, bain $3.33 \%$, vaporisation $1.67 \%$ (Figure 8 ).

On rencontre néanmoins d'autres procédés tels que le lavement, l'inhalation, la voie percutanée, les instillations nasales, oculaires ou auriculaires (Tamboura, Kaboré, \& Yaméogo, 1998).

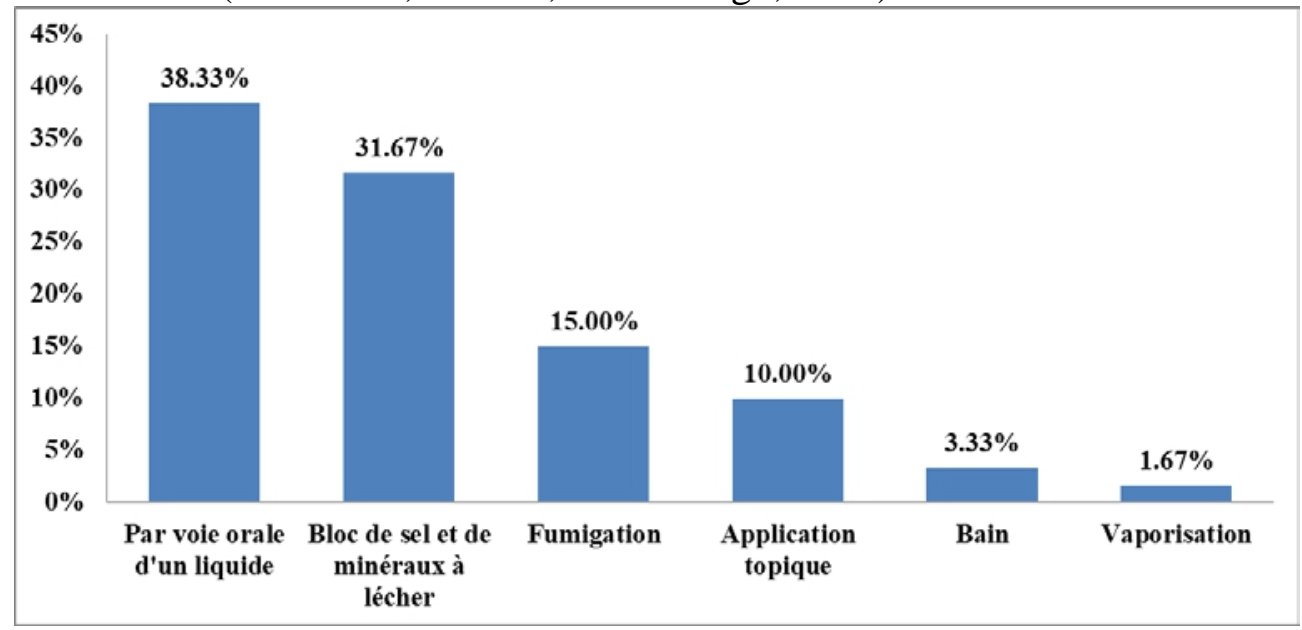

Figure 8 : Répartition de la fréquence des espèces médicinales selon le mode d'administration. 


\section{Efficacité du traitement}

Efficacité du traitement est déclarée selon les informateurs comme sans effet $46.67 \%$ alors ceux qui sont satisfait par les recettes à base de plante médicinale administrer à l'animale d'élevage représentent 53.33\% (Tableau 4).

Selon (Bâ, 1994; Tamboura et al., 1998; Zerbo, 1994) il existe également des maladies bien connues sur les plans cliniques et épidémiologiques, mais pour lesquelles les tradipraticiens affirment ne disposer d'aucun remède.

La confirmation de l'efficacité des extraits de certaines plantes inventoriées au cours des enquêtes vient conforter la connaissance empirique des activités des plantes par les éleveurs. Ceci pourrait s'expliquer par le fait que ces connaissances ont été acquises suite à des expériences répétées sur le terrain. (Ogni et al., 2014)

Tableau 4 : Répartition de la fréquence des espèces médicinales selon leurs efficacités.

\begin{tabular}{|c|c|}
\hline Efficacité du traitement & Pourcentage \\
\hline Aucune & $46,67 \%$ \\
\hline Bien & $30 \%$ \\
\hline Très bien & $23.33 \%$ \\
\hline
\end{tabular}

\section{Disponibilité dans la région}

$51.67 \%$ des enquêteurs confirment que les plantes médicinales sont moins abondantes (Tableau 5). les changements climatiques peuvent engendrer la disparition des espèces végétales et animales.

La biosynthèse des chlorophylles est beaucoup plus inhibée par le froid que par la chaleur (Côme, 1992).

Tableau 5 : Répartition de la fréquence des espèces médicinales selon leurs disponibilités.

\begin{tabular}{|c|c|}
\hline Disponibilité dans la région & Pourcentage \\
\hline Moins disponible & $51.67 \%$ \\
\hline Disponible & $48.33 \%$ \\
\hline
\end{tabular}

\section{AXE 3 : Le traitement des animaux d'élevage Taux d'animaux d'élevage}

Les animaux qui ont été bénéficié d'un traitement traditionnel à base de plante sont les moutons $25 \%$, les poulets $20 \%$, les vaches $10 \%$, alors que le groupe des caprins, ânes, chevaux, oiseaux, chiens, chats, représentent 45\% (Figure 9).

L'aviculture traditionnelle pratiqué depuis très longtemps existe toujours. Il se caractérise par un faible investissement initial, il est présent essentiellement dans les zones rurales, c'est toujours une tradition d'associer l'élevage de volaille aux autres cultures agricoles.

Les ovins et les caprins doivent couvrir les besoins courants en trésorerie familiale. Mais ils contribuent également à la sécurité de 
l'exploitation : en cas de besoin monétaire, pour éviter de toucher à la troupe des reproducteurs bovins, les éleveurs peuvent vendre une partie ou même la totalité des troupeaux de petits ruminants. (Madani, Hubert, Lasseur, \& Guérin, 2001)

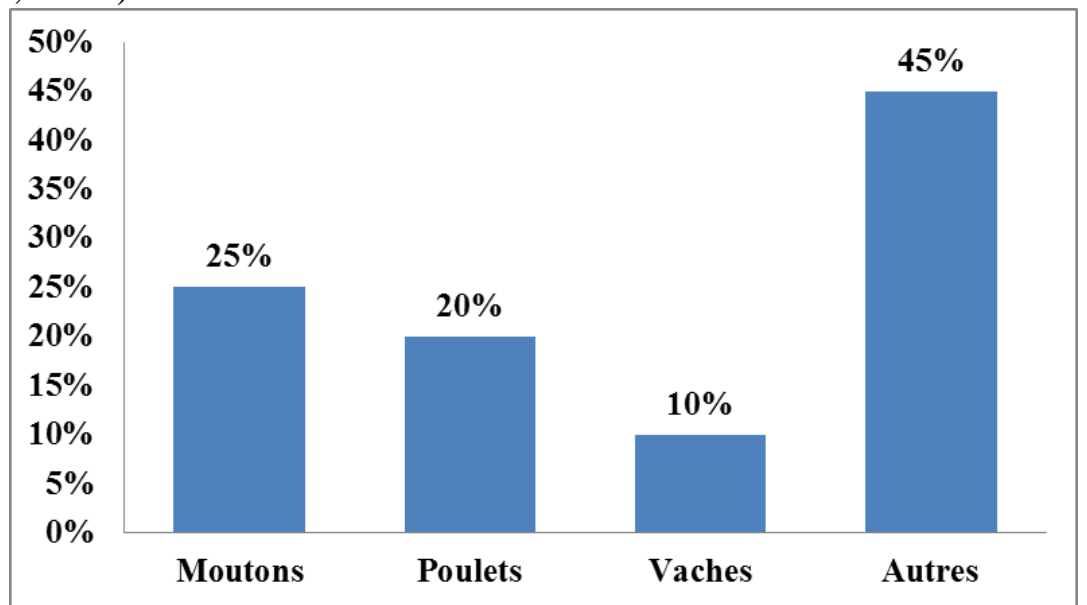

Figure 9 : la fréquence du Taux d’animaux d’élevage.

\section{Les symptômes observés}

Selon les enquêtés les symptômes observés sont classé dans leur importance :

Diarrhées $31.67 \%$, problèmes respiratoires $20 \%$, perte de poids $18.33 \%$, problèmes dermatologique $8.33 \%$, mouvements aléatoires 6.33\% incapacité de marcher 3.33\% autres tel que les blessures 12\% (Figure 10).

Ces résultats sont les mêmes déclaré par Danho et al (2002) dans l'étude d'amélioration de l'environnement sanitaire de la volaille traditionnelle: cas de la cote d'ivoire

Les volailles traditionnelles se nourrissent généralement de détritus et des restes de nourritures de l'alimentation des populations qu'elles trouvent au cours de leur divagation.(Danho, Bodjo, Adon, Kacou, \& CouacyHymann, 2002)

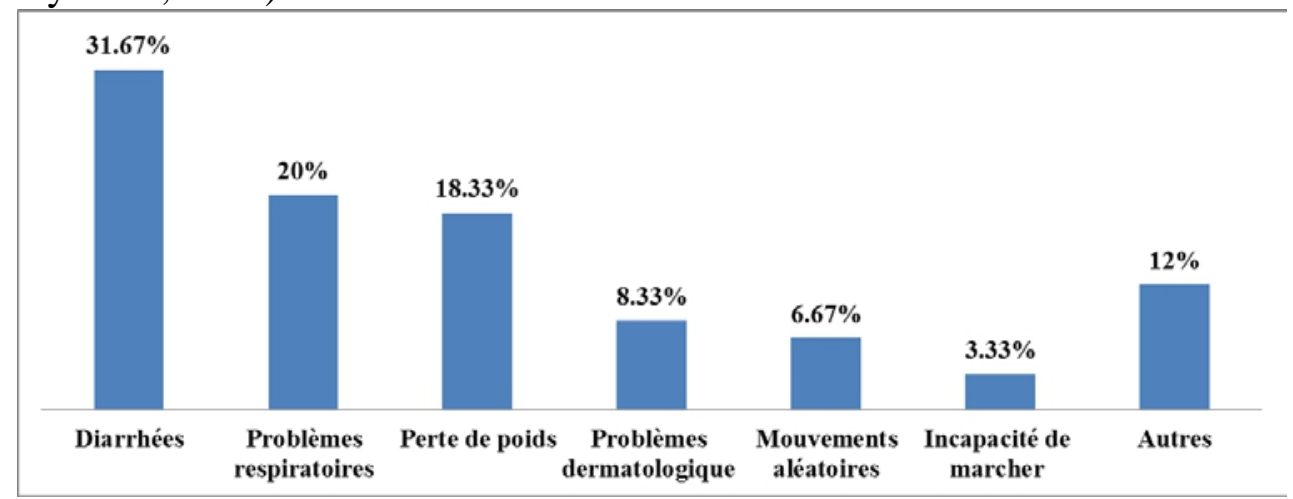

Figure 10 : Les symptômes observés dans un cheptel aviaire. 


\section{Conclusion}

Cette étude a montré que les plantes sont utilisées face à la médecine moderne, malgré que les praticiens sont loin d’être des scientifiques, qui sont pour la plus part des analphabètes avec une moyenne d’âge de 60 ans. Ces pratiques se réalisent à l'aide des organes de sens, entraînant un mauvais pronostique, et le manque des moyens de mesure le volume et le poids en outre les moyens de pronostique pose un problème phytothérapeutique. Non la maitrise des modalités d'administration des médicaments pas les éleveurs, cela et du a l'absence des études sur ces plante ; alors que les médicaments à base de plante administrés peut donner un effet thérapeutique indésirable pour l'animale.

La médecine ethno vétérinaire peut être adoptée dans les cadres de politiques d'élevage des pays en développement tel que le cas Algérie.

Ces résultats confirment que les plantes malgré leurs efficacités de résoudre plusieurs problèmes dans élevage animale dans cette région d'étude ou en Algérie, elles restent coincés par les mauvaises habitudes et les pratiques non hygiéniques.

Ils restent aux études ultérieures à vérifier et confirmer l'efficacité des espèces végétales en aviculture et le contrôle des maladies et des pathologies de poulets.

\section{References:}

Anyinam, C. (1995). Ecology and ethnomedicine: exploring links between current environmental crisis and indigenous medical practices. Social Science \& Medicine, 40(3), 321-329.

Bâ, A. (1994). L'ethnomedecine veterinaire africaine. Paper presented at the Metissages en sante animale de Madagascar a Haiti. Ouagadougou (Burkina Faso). 15-22 Apr 1993.

Benkhnigue, O., Zidane, L., Fadli, M., Elyacoubi, H., Rochdi, A., \& Douira, A. (2010). Etude ethnobotanique des plantes médicinales dans la région de Mechraâ Bel Ksiri (Région du Gharb du Maroc). Acta Botanica Barcinonensia, 53, 191-216.

Bouallala, M., Bradai, L., \& Abid, M. (2014). Diversité et utilisation des plantes spontanées du Sahara septentrional algérien dans la pharmacopée saharienne. Cas de la région du Souf. Revue El-Wahat pour les Recherches et Etudes 7( 2), 18 - 26.

Chehma, A., \& Djebar, M. R. (2008). Les espèces médicinales spontanées du sahara septentrional algérien: distribution spatio-temporelle et étude ethnobotanique. Synthèse: Revue des Sciences et de la Technologie, 17, 3645.

Côme, D. (1992). Les végétaux et le froid: Hermann. 
Danho, T., Bodjo, S., Adon, H., Kacou, A., \& Couacy-Hymann, E. (2002). Amélioration de l'environnement sanitaire de la volaille traditionnelle: cas de la Côte d'Ivoire. Paper presented at the Conference Proceedings]. Characteristics and Parameters of Family Poultry Production in Africa Conference. Available from http://www-naweb. iaea. org/nafa/aph/public/aphpoultry-africa. html.

Daoudi , A., Bachiri , L., Bammou , M., Ibijbijen , J., \& Nassiri , L. (2015). étude ethnobotanique au moyen atlas central. European Scientific Journal, 11(24).

El Hafian, M., Benlandini, N., Elyacoubi, H., Zidane, L., \& Rochdi, A. (2014). Étude floristique et ethnobotanique des plantes médicinales utilisées au niveau de la préfecture d'Agadir-Ida-Outanane (Maroc). Journal of Applied Biosciences, 81(1), 7198-7213.

Hammadi, D., Ahmed, M., Boudjethia, K., Boukhalfa, A., \& Djebli, N. (2015). Ethnomedicinal Survey of Medicinal Plants Used in the Western Region of Algeria. Medicinal \& Aromatic Plants, 5 (1).

Jean, V., \& Jiri, S. (1983). Plantes médicinales. 250 illustrations en couleurs", Larousse ( Larousse ed.). Paris: .

Kahouadji, A. (1986). Recherches floristiques sur le massif montagneux des Beni-Snassene (Maroc oriental). Montpellier 2.

Kpodékon, T., Ogni, C., Dassou, H., Dougnon, T., Boko, C., Koutinhouin, G., . . . Youssao, I. (2015). Dominant viral pathologies in the extensive and semi-intensive animal breeding and their treatment mode in ethno veterinary medicine in Benin. Veterinary World, 8(12), 1424-1434.

Lulekal, E., Asfaw, Z., Kelbessa, E., \& Van Damme, P. (2014). Ethnoveterinary plants of Ankober District, North Shewa Zone, Amhara Region, Ethiopia. Journal of ethnobiology and ethnomedicine, 10(1), 1.

Lyes, \& Kirouani. (2015). Structure et organisation de la filière avicole en Algérie- Cas de la wilaya de Bejaia. El-Bahith Review 15( ), 187-199.

Madani, T., Hubert, B., Lasseur, J., \& Guérin, G. (2001). Association des bovins, des ovins et des caprins dans les élevages de la suberaie algérienne. Cahiers Agricultures, 10(1), 9-18.

McCorkle, C. M. (1986). An introduction to ethnoveterinary research and development: publisher not identified.

Mehdioui, R., \& Kahouadji, A. (2007). Etude ethnobotanique auprès de la population riveraine de la forêt d'Amsittène: cas de la Commune d'Imi n'Tlit (Province d'Essaouira). Bulletin de l'Institut scientifique, Rabat, section Sciences de la vie, 29, 11-20.

Ogni, C., Kpodekon, M., Dassou, H., Boko, C., Koutinhouin, B., Dougnon, J., . . A Akoegninou, A. (2014). Inventaire ethno-pharmacologique des plantes utilisées dans le traitement des pathologies parasitaires dans les élevages 
extensifs et semi-intensifs du Bénin. International Journal of Biological and Chemical Sciences, 8(3), 1089-1102.

SALHI, S., FADLI, M., ZIDANE, L., \& DOUIRA, A. (2010). Etudes floristique et ethnobotanique des plantes médicinales de la ville de Kénitra (Maroc). Lazaroa(31), 133-146.

Tahri, N., El Basti, A., Zidane, L., Rochdi, A., \& Douira, A. (2012). Etude Ethnobotanique Des Plantes Medicinales Dans La Province De Settat (Maroc). Kastamonu Üniversitesi Orman Fakültesi Dergisi, 12(2), 192-208.

Tamboura, H., Kaboré, H., \& Yaméogo, S. M. (1998). Ethnomédecine vétérinaire et pharmacopée traditionnelle dans le plateau central du Burkina Faso: cas de la province du Passoré. Biotechnologie, agronomie, société et environnement, 2(3), 181-191.

Zerbo, K. (1994). Savoirs, savoir-faire, faire savoir et developpement endogene en Afrique. Paper presented at the Metissages en sante animale de Madagascar a Haiti. Ouagadougou (Burkina Faso). 15-22 Apr 1993. 